\title{
Time-Dependent Relationship between the Dorsal Hippocampus and the Prefrontal Cortex in Spatial Memory
}

\author{
Inah Lee ${ }^{1}$ and Raymond P. Kesner ${ }^{2}$ \\ ${ }^{1}$ Departments of Neurobiology and Anatomy, University of Texas Houston Medical School, Houston, Texas 77025, and ${ }^{2}$ Department of Psychology, \\ University of Utah, Salt Lake City, Utah 84112
}

\begin{abstract}
The prefrontal cortex and the dorsal hippocampus have been studied extensively for their significant roles in spatial working memory. A possible time-dependent functional relationship between the prefrontal cortex and the dorsal hippocampus in spatial working memory was tested. A combined lesion and pharmacological inactivation technique targeting both the dorsal hippocampus and the medial prefrontal cortex was used (i.e., axon-sparing lesions of the dorsal hippocampus combined with reversible inactivation of the medial prefrontal cortex, or vice versa, within a subject). A delayed nonmatching-to-place task on a radial eight-arm maze with short-term (i.e., $10 \mathrm{sec}$ ) versus intermediate-term (i.e., $5 \mathrm{~min}$ ) delays was used as a behavioral paradigm. Here we report that the dorsal hippocampus and the medial prefrontal cortex process short-term spatial memory in parallel, serving as a compensatory mechanism for each other. The role of the dorsal hippocampus, however, becomes highlighted as the time-window for memory (i.e., delay) shifts from short-term to a delay period (i.e., intermediate-term) exceeding the short-term range. The results indicate that the time window of memory is a key factor in dissociating multiple memory systems.
\end{abstract}

Key words: dorsal hippocampus; medial prefrontal cortex; spatial working memory; ibotenic acid; quinolinic acid; short-term memory; intermediate-term memory

\section{Introduction}

It is widely accepted that damage of the hippocampal system typically produces an episodic or spatial memory loss (Scoville and Milner, 1957; Jarrard, 1993; Eichenbaum, 2000). However, a large number of studies indicate that short-term memory is spared, whereas intermediate-term memory is relatively impaired with damage to the hippocampal system in both humans (Scoville and Milner, 1957; Eichenbaum et al., 1994; Holdstock et al., 1995; Eichenbaum, 2000; Kesner and Hopkins, 2001) and animals (Mishkin, 1978; Kesner and Novak, 1982; Kesner and Adelstein, 1989; Winocur, 1992; Jarrard, 1993; Alvarez et al., 1994; Eichenbaum et al., 1994; Steele and Morris, 1999; Eichenbaum, 2000; Clark et al., 2001). The relatively intact short-term memory with hippocampal dysfunction in the above literature, however, is somewhat in contrast to the computational models of the hippocampus, because those models suggest that the hippocampus mediates shortterm memory mainly by virtue of the recurrent collaterals in CA3 brain region (Granger et al., 1996; Wiebe et al., 1997; Kesner and Rolls, 2001).

To address the null effects of hippocampal damage on shortterm memory observed in previous literature, neocortical contributions have been suggested (Eichenbaum et al., 1994; Eichenbaum, 2000). Among neocortical areas, there is good evidence that the prefrontal cortex (PFC) plays an important role in

\footnotetext{
Received Sept. 9, 2002; revised Nov. 13, 2002; accepted Nov. 22, 2002.

The work was supported by Human Frontiers Science Program Grant RG0110/1998B and National Science Foundation Grant IBN 9817583

Correspondence should be addressed to Raymond P. Kesner, Department of Psychology, University of Utah, 380 S 1530E Room 502, Salt Lake City, UT 84112. E-mail: rpkesner@behsci.utah.edu.

Copyright $\odot 2003$ Society for Neuroscience $\quad 0270-6474 / 03 / 231517-07 \$ 15.00 / 0$
}

short-term memory, especially in a delayed matching- or nonmatching-to-sample task in which a correct choice response for a stimulus (e.g., object or spatial location) is required after a delay period (Delatour and Gisquet-Verrier, 1996; Floresco et al., 1997; Porter et al., 2000; Fuster, 2001; Izaki et al., 2001). Strong electrophysiological correlates can be identified during a shortterm delay period within PFC in nonhuman primates in delayedchoice tasks (Rainer et al., 1998; Constantinidis et al., 2001). The rodent medial PFC ( $\mathrm{mPFC}$ ) also plays an equivalent role in tasks in which a delayed choice response to stimuli is required and lesions of mPFC impair performance in delayed choice tasks (Shaw and Aggleton, 1993; Delatour and Gisquet-Verrier, 1996).

The main goal of the current study was to examine the interactions between PFC and the dorsal hippocampus in controlling short-term and intermediate-term working memory for spatial information. A double-manipulation technique consisting of axon-sparing lesion and reversible inactivation within subjects was used in a delayed nonmatching-to-place task on a radial eight-arm maze. Our prediction is that the dorsal hippocampus may play a more important role in intermediate-term memory compared with $\mathrm{mPFC}$ based on the previous literature (Scoville and Milner, 1957; Mishkin, 1978; Kesner and Novak, 1982; Kesner and Adelstein, 1989; Winocur, 1992; Jarrard, 1993; Alvarez et al., 1994; Eichenbaum et al., 1994; Holdstock et al., 1995; Steele and Morris, 1999; Eichenbaum, 2000; Porter et al., 2000; Clark et al., 2001; Fuster, 2001; Izaki et al., 2001; Kesner and Hopkins, 2001). However, the dorsal hippocampus or mPFC may take over the function of short-term spatial working memory when the other structure is unavailable, because previous literature suggests that both the PFC and the hippocampus are involved in spatial working memory with short-term delays (Hampson et al., 1999; Fuster, 2001). 


\section{Materials and Methods}

\section{Subjects}

Twenty-two male Long-Evans rats (260-400 gm) were housed individually in standard rodent cages. They were maintained on a $12 \mathrm{hr}$ light/ dark cycle. For behavioral testing, each rat was initially food deprived to $80 \%$ of its free-feeding weight and allowed access to water ad libitum. All behavioral experiments were performed during the light phase of the light/dark cycle.

\section{Behavioral apparatus}

A radial eight-arm maze was used throughout the entire experiment. The maze was surrounded by $8-10$ distinct visual cues that were present mostly near the walls of the room. The maze had a center platform with a diameter of $40 \mathrm{~cm}$ and eight arms of $60 \mathrm{~cm}$ long and $9 \mathrm{~cm}$ wide. It also had Plexiglas walls $5.7 \mathrm{~cm}$ in height for each arm. All of the arms of the maze had food wells $2.5 \mathrm{~cm}$ in diameter and $1.5 \mathrm{~cm}$ deep at the distal ends. Rewards (Froot Loops cereal; Kellogg, Battle Creek, MI) were placed in these wells. The center platform of the maze was surrounded by walls and doors made of transparent Plexiglas, so that rats could clearly see extramaze cues. Each door could be opened from outside of the testing room. Above the center platform, there was a cylindrical plastic bucket (38 cm in diameter and $75 \mathrm{~cm}$ in height) that could be lowered from outside of the testing room. The bucket was opaque and the inner wall was painted white, so that rats could not detect any local cues on the inner bucket surface. The bucket, when lowered down to the center platform, completely prevented rats from viewing extramaze cues.

\section{Procedures}

Behavioral pretraining. Male Long-Evans rats $(n=22)$ were trained on a radial eight-arm maze using a delayed-nonmatching-to-place (DNMP) paradigm (Lee and Kesner, 2002). After visiting an arm (i.e., study arm) for a reward, each rat was confined in a bucket on the center platform for a $10 \mathrm{sec}$ delay period during which doors for both an adjacent arm (i.e., choice arm) and the visited study arm were opened. The rat had to choose the unvisited choice arm to receive a reward. If the rat selected the study arm again either by completely traversing the study arm to the end or when both of the rear paws touched the study arm, it was recorded as an error. On each trial, a study arm was randomly selected and a choice arm was always an adjacent arm on either side (randomly chosen) of the study arm. The purpose of this design was to make two arms (study and choice arms) equally available at the time of the choice phase by preventing the situation in which the rat might easily avoid choosing a study arm by remembering the direction information of the study arm rather than the spatial cues. Eight trials per day were given with an intertrial interval of 20 sec for six blocks (two days were grouped as one block to reduce possible daily variability from drug applications). After pretraining to a criterion ( $>95 \%$ correct choices), each rat was given surgery under Nembutal anesthesia for a cannula implant and a lesion.

Surgery. After behavioral pretraining rats $[\mathrm{HP} / \mathrm{LES}+\mathrm{mPFC} / \mathrm{drug}$ group (the group with dorsal hippocampal lesions and drug injections into $\mathrm{mPFC}) ; n=6$ ] were bilaterally implanted with guide cannulas (22 gauge) coupled with stylets ( 28 gauge, $1 \mathrm{~mm}$ protrusion) in $\mathrm{mPFC}$ (i.e., prelimbic and infralimbic areas) in addition to bilateral lesions of the dorsal hippocampus with multiple injections of ibotenic acid $(0.6 \mathrm{mg} / \mathrm{ml}$; $0.2-0.3 \mu \mathrm{l} /$ site) with a $10 \mu \mathrm{l}$ Hamilton syringe (Hamilton, Reno, NV) driven by a microinfusion pump (Cole-Parmer, Vernon Hills, IL). Only the dorsal hippocampus, not the ventral hippocampus, was targeted in our study on the basis of its well known involvement in spatial learning and memory (Moser et al., 993; Moser and Moser, 1998). The role of the dorsal hippocampus in the current spatial working memory task was confirmed previously in our laboratory (Lee and Kesner, 2002).

Each animal was injected with atropine sulfate $(0.2 \mathrm{mg} / \mathrm{kg}$, i.p. $)$ and deeply anesthetized with sodium pentobarbital (Nembutal; $60 \mathrm{mg} / \mathrm{kg}$, i.p.). The animal was placed in a stereotaxic instrument (David Kopf Instruments, Tujunga, CA), and an incision was made along the midline of the scalp. The skull was exposed, and the instrument was adjusted to ensure a flat skull surface. Small burr holes were drilled in the skull using the following coordinates: (1) hippocampal lesions: (a) $2.8 \mathrm{~mm}$ posterior to bregma, $1.6 \mathrm{~mm}$ lateral to midline, and $3.0 \mathrm{~mm}$ ventral from dura, (b)
$3.3 \mathrm{~mm}$ posterior to bregma, $1.8 \mathrm{~mm}$ lateral to midline, and $2.8 \mathrm{~mm}$ ventral from dura, and (c) $4.1 \mathrm{~mm}$ posterior to bregma, $2.6 \mathrm{~mm}$ lateral to midline, and $2.8 \mathrm{~mm}$ ventral from dura; (2) mPFC cannulas: $3.0 \mathrm{~mm}$ anterior to bregma, $2.0 \mathrm{~mm}$ lateral to midline, and $4.6 \mathrm{~mm}$ ventral from dura with $25^{\circ}$ angle from a vertical midline. A control group [HP/ $\mathrm{CT}+\mathrm{mPFC} /$ drug group (the group with sham lesions in the dorsal hippocampus and drug injections into $\mathrm{mPFC}$ ); $n=5$ ] was generated using the same procedures, except that saline was injected in the dorsal hippocampus instead of ibotenic acid.

The other group [HP/drug $+\mathrm{mPFC} / \mathrm{LES}$ group (the group with drug injections into the dorsal hippocampus and mPFC lesions); $n=6$ ] was implanted bilaterally with cannulas in the dorsal hippocampus and bilateral lesions in $\mathrm{mPFC}$ with quinolinic acid (0.09 $\mathrm{M}, 0.3-0.5 \mu \mathrm{l} / \mathrm{site}$; Research Biochemicals, Natick, MA). The following coordinates were used: (1) hippocampal cannulas: $3.6 \mathrm{~mm}$ posterior to bregma, $2.4 \mathrm{~mm}$ lateral to midline, and 2.8 ventral from dura; (2) mPFC lesions: (a) 3.5 $\mathrm{mm}$ anterior from bregma, $0.6 \mathrm{~mm}$ lateral from midline, and $3.8 \mathrm{~mm}$ ventral from dura, and (b) $2.5 \mathrm{~mm}$ anterior from bregma, $0.6 \mathrm{~mm}$ lateral from midline, and 4.0 ventral from dura. The ventral coordinate was used to adjust the depth of the tips of stylets coupled with the guide. A control group $[\mathrm{HP} / \mathrm{drug}+\mathrm{mPFC} / \mathrm{CT}$ group (the group with drug injections into the dorsal hippocampus and control lesions in $\mathrm{mPFC}$ ); $n=5$ ] was given the same surgical procedures, except that saline was injected into mPFC. PBS, pH 7.4, or muscimol (MUS) $(0.5 \mu \mathrm{g} / 0.5 \mu \mathrm{l})$ was injected for all groups during behavioral testing. All protocols conformed to the NIH Guide for the Care and Use of Laboratory Animals and the Institutional Animal Care and Use Committee at the University of Utah.

Behavioral postsurgery testing. After a recovery period, the rats were retested in the same room used for pretraining with two blocks of PBS injection into either the mPFC or the dorsal hippocampus for a total of 32 trials (i.e., $4 \mathrm{~d}$ ) using $10 \mathrm{sec}$ delays. Then, the animals were tested for $4 \mathrm{~d}$ with PBS injections using a variable delay DNMP paradigm in which intermediate delays (i.e., $5 \mathrm{~min}$; four trials) were randomly intermixed with the original short delays (i.e., $10 \mathrm{sec}$; four trials) in the session of a given day. Finally, the rats were given 4 more days of the same variable delay DNMP paradigm with muscimol injections.

Intracranial microinjection. The drug injection protocol used for behavioral testing was as follows. Muscimol, a GABA agonist, was dissolved in PBS in final concentration of $0.5 \mu \mathrm{g} / 0.5 \mu \mathrm{l}$. Muscimol was chosen as an inactivating agent over other short-lasting inactivating agents [e.g., lidocaine (Martin, 1991)] considering the relatively long testing period of our behavioral paradigm ( $\sim 30 \mathrm{~min}$, especially in the $10 \mathrm{sec}$ vs $5 \mathrm{~min}$ paradigm). Either muscimol or PBS was injected bilaterally via an injection needle (28 gauge) $30 \mathrm{~min}$ before the behavioral experiment of each day. The injection quantity was $0.5 \mu \mathrm{l} / \mathrm{side}$, and the injection rate was at 0.1 $\mu \mathrm{l} / \mathrm{min}$. The injection was made with a $10 \mu \mathrm{l}$ Hamilton syringe driven by a microinfusion pump. The injection needle was left in place for $1 \mathrm{~min}$ after the injection. The rat was then returned to its home cage, and any abnormality in movement from the drug injection was carefully examined for $30 \mathrm{~min}$ before the rat was placed on the maze.

\section{Histology}

Histological verification of cannula positions and lesions was performed after the completion of all behavioral experiments. Rats received a lethal dose of sodium pentobarbital, followed by a transcardial infusion of $0.9 \%$ saline and a $10 \%$ formaldehyde solution. Each brain was stored in a $10 \%$ formalin- $30 \%$ sucrose solution at $4^{\circ} \mathrm{C}$ for $72 \mathrm{hr}$. The brains were frozen, cut in coronal sections on a cryostat, and stained with cresyl violet.

\section{Results}

Histology

Figure 1 illustrates the cannula positions and the extent of neurotoxic lesions. Figure $1 a$ shows that all of the cannulas were implanted in the $\mathrm{MPFC}$ region in rats included in final behavioral data analysis. It also shows that ibotenic acid injected into the dorsal hippocampus was effective in eliminating most of the dorsal hippocampus, although, in some cases, minor overlying cortices were inevitably disrupted mainly from mechanical damage 
a

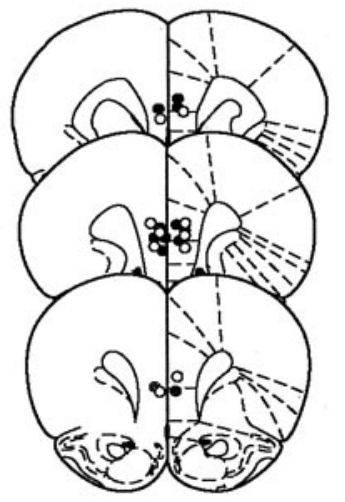

2.2

2.7

3.2

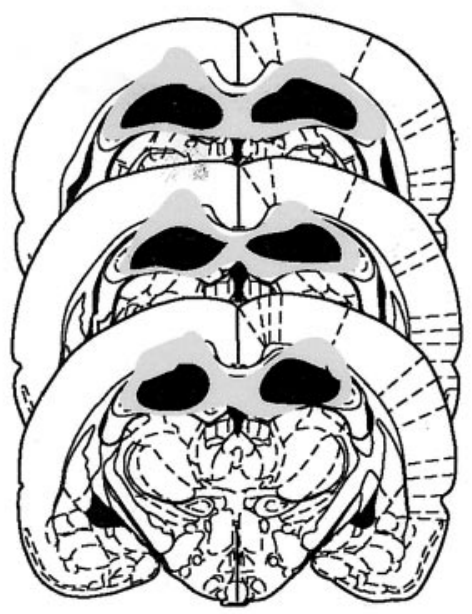

$-4.1$

b

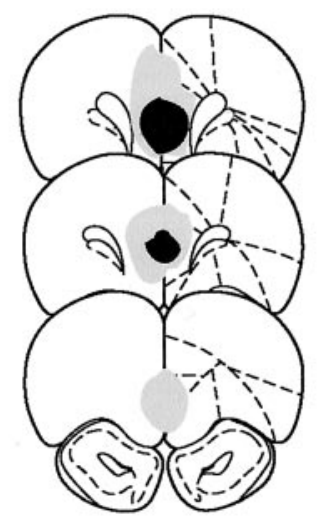

3.2

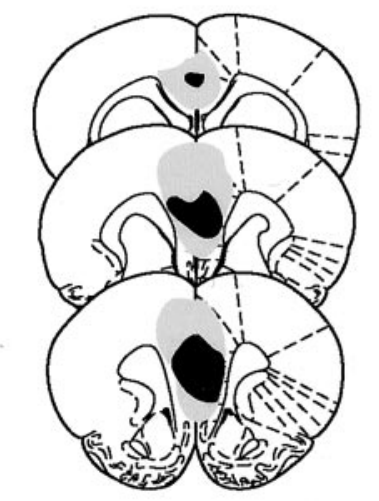

1.7

2.2

2.7

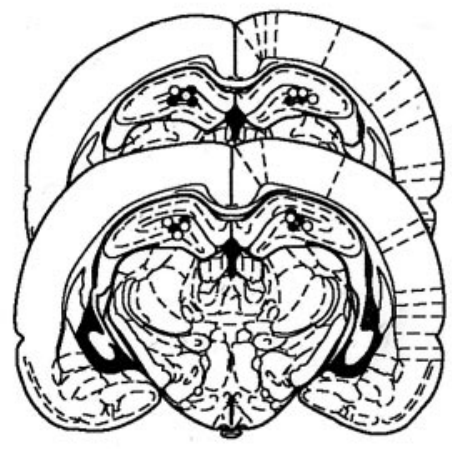

$-3.6$

Figure 1. Illustration of the positions of the cannulas and the extent of damage by excitotoxic lesions in a series of sections of mPFC and dorsal hippocampus. $a$, Histological verification of the $\mathrm{HP} / \mathrm{LES}$ (or HP/CT) + mPFC/drug group. Cannula positions are marked in mPFC (O, HP/CT + mPFC/drug group;, $\mathrm{HP} / \mathrm{LES}+\mathrm{mPFC} /$ drug group). The largest and the smallest tissue damage produced by ibotenic acid in the dorsal hippocampus (i.e., HP/LES $+\mathrm{mPFC} /$ drug group) are shown by gray and black, respectively. $b$, Histological verifications of the $\mathrm{mPFC} / \mathrm{LES}$ (or $\mathrm{mPFC/CT)}+\mathrm{HP} / \mathrm{drug}$ group. Cannula positions are shown in the dorsal hippocampus (, $\mathrm{mPFC} / \mathrm{CT}+\mathrm{HP} /$ drug group;, $\mathrm{mPFC} / \mathrm{LES}+\mathrm{HP} /$ drug group). The largest and the smallest tissue damage produced by quinolinic acid in $\mathrm{mPFC}$ are shown by gray and black, respectively. The anteroposterior stereotaxic coordinates for the sections were shown by the numbers beside the sections (in millimeters) (modified from Paxinos and Watson, 1997).

by the injection needle. However, such cortical damage was also observed in the control lesion groups, and, during histological verification, most overlying cortices beyond the injection sites were intact. No extrahippocampal damage (e.g., subiculum and entorhinal cortex) was observed as a result of axon-sparing lesions. Figure $1 b$ shows that quinolinic acid injected into mPFC region eliminated cells mostly in the prelimbic and infralimbic areas. In some cases, ventromedial orbital areas as well as the anterior cingulate region dorsal to the $\mathrm{mPFC}$ were affected as reported previously (Ragozzino et al., 2002). Figure $1 b$ also illustrates histologically verified cannula positions in the dorsal hippocampi. Most cannulas were placed in the upper dentate/hilar area to maximize the spread of muscimol to the entire dorsal hippocampus.

\section{Behavior}

Spatial memory with short-term delays

Rats had to learn to choose between a visited arm (study arm) and an unvisited arm (choice arm) with a short-term delay ( $10 \mathrm{sec})$ imposed between the two arms of a radial eight-arm maze. Groups of rats were given different combinations of neurotoxic (or sham) lesions and cannulas implanted in the dorsal hippocampus and mPFC (i.e., HP/CT+mPFC/drug, HP/drug $+\mathrm{mPFC} / \mathrm{CT}, \mathrm{HP} / \mathrm{LES}+\mathrm{mPFC} /$ drug, and HP/drug + mPFC/LES) (Fig. 1).

When the rats were tested for postsurgery performance in the same task with PBS, pH 7.4, injections, both the HP/LES + mPFC/ PBS (the group with dorsal hippocampal lesions and PBS injections into the $\mathrm{mPFC}$ ) and the HP/PBS $+\mathrm{mPFC} / \mathrm{LES}$ (the group with PBS injections into the dorsal hippocampus and $\mathrm{MPFC}$ lesions) groups showed impaired performance (Fig. 2a) during the first block (i.e., one block is $2 \mathrm{~d}$ of eight trials per day) compared with the CT group [data from the HP/CT+mPFC/PBS (the group with control lesions in the hippocampus and PBS injections into the mPFC) and the HP/PBS $+\mathrm{mPFC} / \mathrm{CT}$ (the group with $\mathrm{PBS}$ injections into the hippocampus and control lesions in the $\mathrm{mPFC}$ ) groups were combined into one control group, CT, because of a lack of significant difference between the two groups $(p>0.5)]$. The deficit in performance in both groups, however, recovered to control levels by the second block. An ANOVA with a repeated-measures design showed a significant effect of groups $\left(F_{(2,19)}=20.5 ; p<0.001\right)$. There were also significant effects of blocks $\left(F_{(1,19)}=35.8 ; p<0.001\right)$ and the interaction between groups and blocks $\left(F_{(2,19)}=5.8 ; p<0.05\right)$. A post hoc analysis [Tukey's honestly significant difference (HSD)] demonstrated 

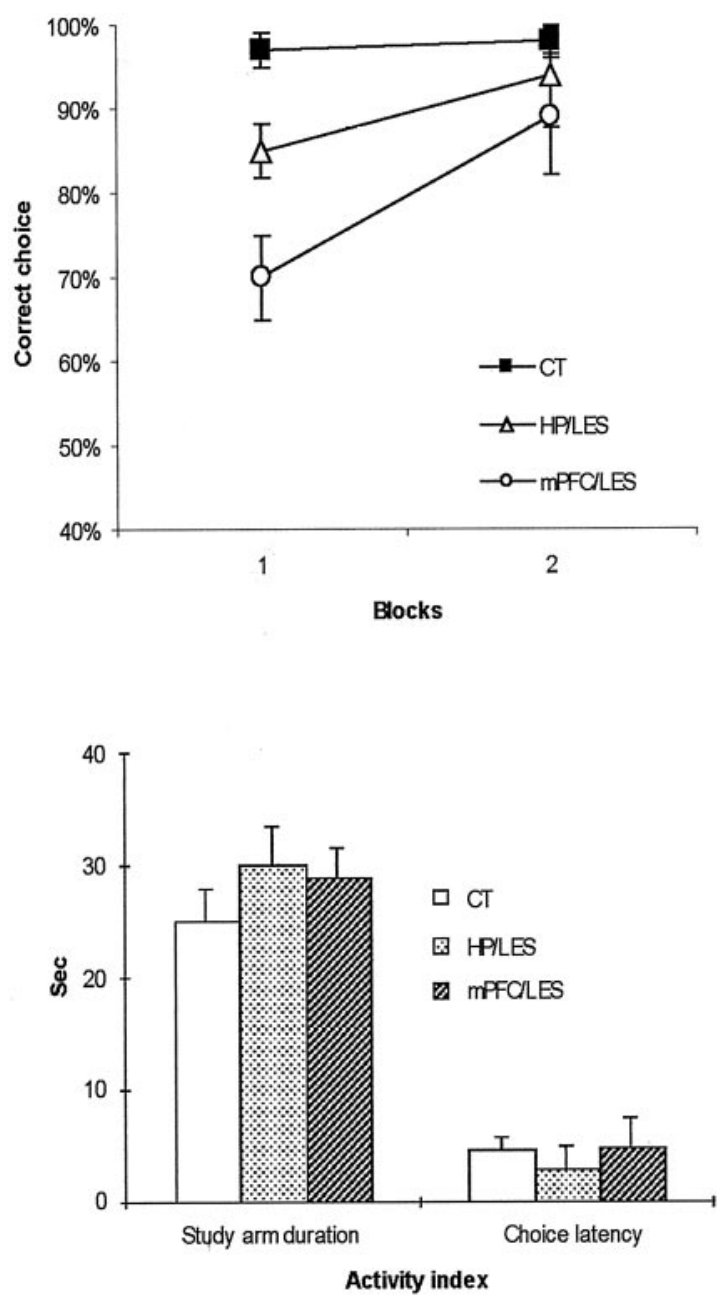

Figure 2. Postsurgery performance in a short-term spatial water maze task. $a$, Choice accuracy for two blocks with PBS injections into either the dorsal hippocampus or mPFC after surgery ( 1 block is $2 \mathrm{~d}$ of 8 trials per day). Note the initial deficit in both lesion groups (but more severely in the $\mathrm{mPFC} / \mathrm{LES}$ group) in block 1 yet with improvement in block 2.b. Average activity levels of different lesion groups during postsurgery performance in the spatial working memory task (Study arm duration, the time spent on a study arm; Choice latency, the latency to reach the end of a choice arm from the center platform). There were no significant differences among the groups. CT, Control group; HP/LES, the group with dorsal hippocampal lesions and PBS injections into $\mathrm{mPFC} ; \mathrm{mPFC} / \mathrm{LES}$, the group with $\mathrm{mPFC}$ lesions and PBS injections into the dorsal hippocampus.

that, only in block 1, both the HP/LES + mPFC/PBS and the HP/ $\mathrm{PBS}+\mathrm{mPFC} / \mathrm{LES}$ groups were significantly impaired in performance compared with the CT group ( $p$ values of $<0.001$ ) with no significant difference between the two groups ( $p$ values of $>0.1$ ). The time spent on the study arm (i.e., study-arm duration) was recorded. The latency to obtain the reward at the end of the choice arm (i.e., choice latency) was also measured. Those activity measures (i.e., study-arm duration and choice latency) (Fig. $2 b$ ) showed no significant difference among the groups ( $p$ values of $>0.1$ for both measures).

Spatial memory with intermediate-term delays

To test the time-dependent functional relationship between mPFC and the dorsal hippocampus, four intermediate-delay (i.e., $5 \mathrm{~min}$ ) trials were randomly intermixed with four original short-delay (i.e., $10 \mathrm{sec}$ ) trials, thus forming a block of eight trials of variable delays per day. All of the groups were first injected with PBS during two blocks of testing (i.e., $4 \mathrm{~d}$ ). Then, they were
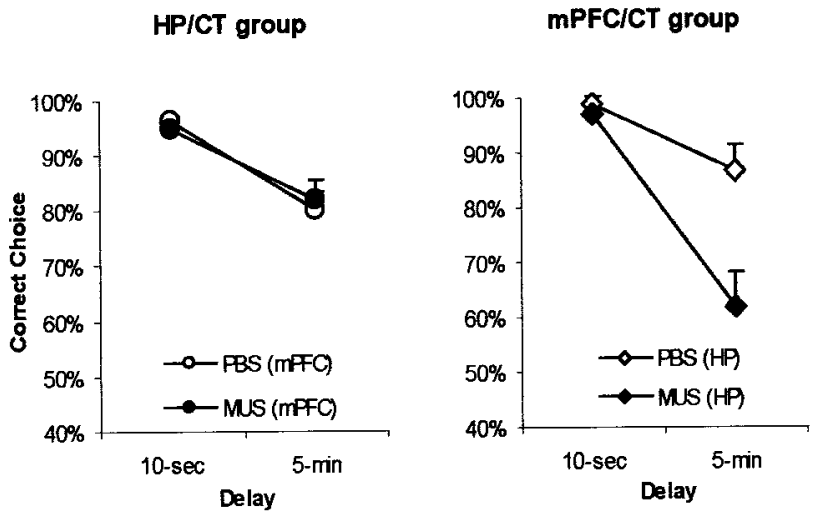

HPILES group
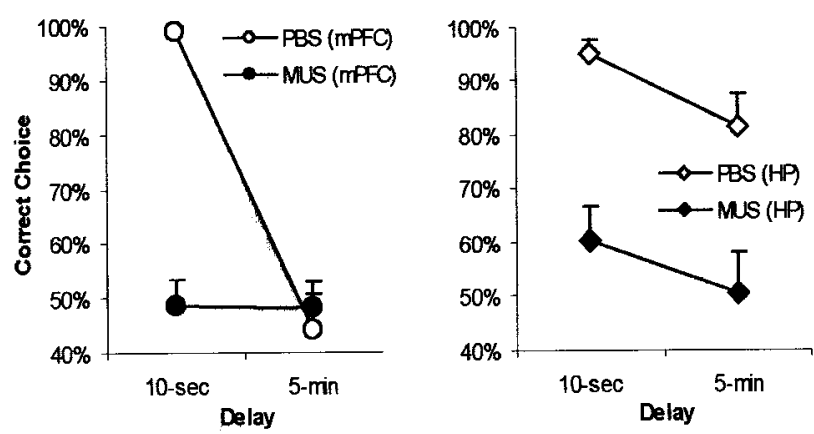

Figure 3. Choice accuracy in different lesion groups after the injection of PBS or MUS in 10 sec or 5 min delay trials. $a$, The hippocampal control lesion group $(H P / C T)$ exhibited no difference in choice accuracy between the conditions with MUS and PBS injections into $\mathrm{mPFC} . b$, The $\mathrm{mPFC}$ control lesion group ( $\mathrm{mPFC} / \mathrm{CT}$ ) showed lower choice accuracy only in $5 \mathrm{~min}$ delay trials with MUS injection into the dorsal hippocampus compared with PBS injection. c, Compared with PBS injection, the hippocampal lesion group (HP/LES) showed a marked deficit in choice accuracy only in 10 sec delay trials with MUS injection into MPFC. Both PBS and MUS injections disrupted performance in 5 min delay trials in the hippocampal lesion group. $d$, The $\mathrm{mPFC}$ lesion group ( $\mathrm{mPFC} / L E S$ ) was impaired in both delay conditions (i.e., 10 sec and $5 \mathrm{~min}$ ) when MUS was injected into the dorsal hippocampus compared with PBS injection.

tested with MUS injections for two more blocks. A dynamic interaction between mPFC and the dorsal hippocampus was observed as a result (Fig. 3). The hippocampal control lesion group (HP/CT) exhibited no difference in choice accuracy between the PBS and MUS injections into mPFC (Fig. 3a). The mPFC control lesion group ( $\mathrm{mPFC} / \mathrm{CT}$ ) showed lower choice accuracy only in 5 min delay trials when MUS was injected into the dorsal hippocampus compared with PBS injections (Fig. 3b). Compared with PBS injections, the hippocampal lesion group (HP/LES) showed a marked deficit in choice accuracy only in $10 \mathrm{sec}$ delay trials with MUS injections into mPFC, whereas both PBS and MUS injections disrupted performance in 5 min delay trials with hippocampal lesions (Fig. $3 c$ ). The mPFC lesion group (mPFC/ LES) was impaired in both delay conditions (i.e., $10 \mathrm{sec}$ and 5 min) when MUS was injected into the dorsal hippocampus compared with PBS injections (Fig. 3d). An ANOVA performed with groups as a between-subject variable and both drugs and delays as two within-subject variables revealed significant effects of groups $\left(F_{(3,18)}=106.2 ; p<0.001\right)$, drugs $\left(F_{(1,18)}=272.1 ; p<0.001\right)$, and delays $\left(F_{(1,18)}=269.7 ; p<0.001\right)$. There were significant interaction effects between groups and within-subjects variables: groups $\times$ drugs, $F_{(3,18)}=44.5, p<0.001$; groups $\times$ delays, 


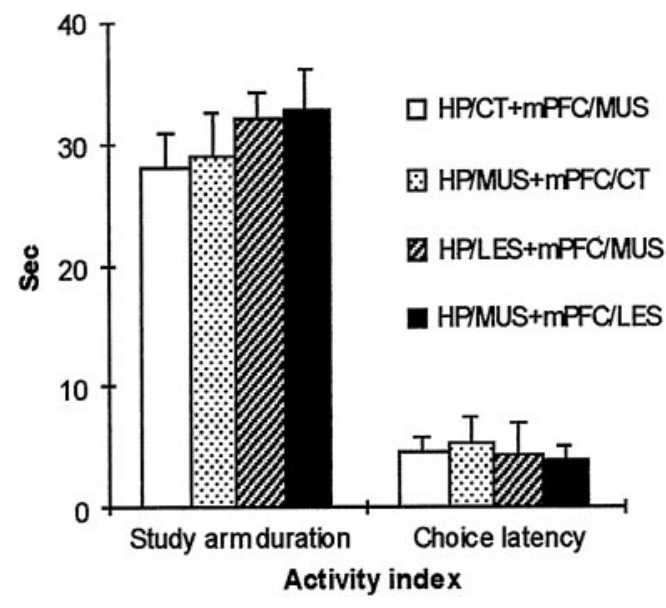

Figure 4. Average activity levels for different groups during 10 sec and 5 min delay trials (Study arm duration, the time spent on a study arm; Choice latency, the latency to reach the end of a choice arm from the center platform). No significant differences were found among the groups when MUS was injected.

$\left.F_{(3,18)}=9.4, p<0.001\right)$; and groups $\times$ drugs $\times$ delays, $F_{(3,18)}=$ $32.1, p<0.001)$.

An additional post hoc analysis (Tukey's HSD) demonstrated that the HP/LES + mPFC/PBS group (Fig. $3 c$ ) was significantly different in choice accuracy in 5 min delay trials from the other PBS-injected groups (i.e., HP/CT $+\mathrm{mPFC} / \mathrm{PBS}, \mathrm{HP} / \mathrm{PBS}+\mathrm{mPFC} / \mathrm{CT}$, and $\mathrm{HP} /$ $\mathrm{PBS}+\mathrm{mPFC} / \mathrm{LES}$ ) ( $p$ values of $<0.001$ ). Both double-inactivation groups for the dorsal hippocampus and mPFC (i.e., the HP/ LES $+\mathrm{mPFC} / \mathrm{MUS}$ and the HP/MUS + mPFC/LES in Fig. 3, $c$ and $d$, respectively) were significantly different in $10 \mathrm{sec}$ delay trials from both single-inactivation groups with sham lesions (i.e., the HP/ $\mathrm{CT}+\mathrm{mPFC} / \mathrm{MUS}$ and the HP/MUS $+\mathrm{mPFC} / \mathrm{CT})$ in choice accuracy ( $p$ values of $<0.001$ ). In 5 min delay trials, the $\mathrm{HP} / \mathrm{CT}+\mathrm{mPFC} /$ MUS group (Fig. $3 a$ ) showed significantly higher choice accuracy compared with the HP/MUS $+\mathrm{mPFC} / \mathrm{CT}$ group $(p<0.01)$ and the other two hippocampal inactivation groups (i.e., $\mathrm{HP} / \mathrm{LES}+\mathrm{mPFC} /$ MUS and HP/MUS + mPFC/LES; $p$ values of $<0.001)$. The activity measures showed no significant difference among the groups in the MUS-injection conditions ( $p$ values of $>0.1$ in both measures) (Fig. 4). The results showed that short-term memory (i.e., $10 \mathrm{sec}$ ) was intact only when either the dorsal hippocampus or mPFC was allowed to function normally. Intermediate-term memory (i.e., 5 min), however, was severely impaired whenever the dorsal hippocampus was inactivated, regardless of $\mathrm{mPFC}$ inactivation.

\section{Discussion}

Our results showed that inactivating either mPFC or the dorsal hippocampus produced an initial deficit in short-term (i.e., 10 sec) spatial working memory, which eventually improved over time to a normal level of performance. This suggests that inactivating one of these structures initially affected the short-term working memory system, but a compensatory adjustment was made to achieve normal performance. Therefore, it seems that the dorsal hippocampus and mPFC normally process short-term spatial working memory in parallel, which may explain the null effect of hippocampal damage on short-term or immediate memory in the previous literature (Scoville and Milner, 1957; Mishkin, 1978; Kesner and Novak, 1982; Kesner and Adelstein, 1989; Winocur, 1992; Jarrard, 1993; Alvarez et al., 1994; Eichenbaum et al., 1994; Holdstock et al., 1995; Steele and Morris, 1999; Eichenbaum, 2000; Porter et al., 2000; Clark et al., 2001; Kesner and Hopkins, 2001). The data also support, based on computa- tional models, a proposed role for the dorsal hippocampus in short-term memory (Granger et al., 1996; Wiebe et al., 1997; Kesner and Rolls, 2001). After the system becomes stabilized, presumably as a result of a compensatory mechanism in the absence of one structure, this compensatory structure might be sufficient for normal performance when information needs to be stored for only seconds (Fig. 2a). Inactivating both regions at the same time, however, resulted in a severe impairment of shortterm spatial working memory, suggesting that one of the structures needs to function properly for intact processing of shortterm spatial working memory. Importantly, the dorsal hippocampus became a necessary structure for spatial working memory after an intermediate-term delay (i.e., $5 \mathrm{~min}$ ), whereas mPFC failed to show compensation for this time window (Fig. 3), suggesting a unique function for the dorsal hippocampus in the time range longer than short term (Scoville and Milner, 1957; Mishkin, 1978; Kesner and Novak, 1982; Kesner and Adelstein, 1989; Winocur, 1992; Jarrard, 1993; Alvarez et al., 1994; Eichenbaum et al., 1994; Holdstock et al., 1995; Steele and Morris, 1999; Eichenbaum, 2000; Porter et al., 2000; Clark et al., 2001; Kesner and Hopkins, 2001).

Memory appears to be the product of dynamic interactions among multiple systems in the brain, and the interaction between the hippocampal and neocortical systems has turned into a topic of importance in studying hippocampal function in memory (Mishkin and Appenzeller, 1987; Squire, 1992; Eichenbaum et al., 1994; Nadel, 1995; Eichenbaum, 2000; Maguire et al., 2000). Although the concept of interaction among multiple brain systems has well been accepted (Nadel et al., 2000; Kim and Baxter, 2001), the mnemonic constraints that control the nature of interaction among those systems are primarily unknown. The current results suggest that the time window of memory (i.e., intermediate-term delay) is a critical factor in dissociating the function of the dorsal hippocampus from that of $\mathrm{mPFC}$ in a delayed choice task. The initial deficits exhibited in both HP/LES + mPFC/PBS and HP/ $\mathrm{PBS}+\mathrm{mPFC} / \mathrm{LES}$ groups suggest that normally there is parallel processing between the dorsal hippocampus and $\mathrm{MPFC}$ for spatial working memory that operates within a short-term range. However, the dorsal hippocampus becomes more essential once the critical time window entails spatial working memory for a period exceeding a short-term range. There are several possibilities that may explain this delay-dependent function toward the dorsal hippocampus for spatial working memory. That is, $\mathrm{mPFC}$ may not have a suitable neural architecture to hold spatial items for more than several seconds. The recurrent networks within the dorsal hippocampus (e.g., CA3), with the aid of the hippocampal-parahippocampal loop, may be better suited to circulate spatial information for a longer time period compared with mPFC. Therefore, the dorsal hippocampus and mPFC might process spatial working memory in parallel initially for a short-term period, but as soon as the system detects that the delay period is prolonged from short-term to intermediate-term (or long-term), hippocampal memory may become essential, demonstrating more persistence than mPFC memory. Alternatively, mPFC per se may not maintain spatial items (Rowe et al., 2000) but has access to multiple brain regions. The rats with hippocampal lesions might still be able to perform well on short-term delay trials, because other associational sensory cortices than the dorsal hippocampus could still provide information on sensory cues to $\mathrm{mPFC}$ for a short-term delay period. However, this information access hypothesis for $\mathrm{mPFC}$ does not seem to be universally applicable, because an mPFC inactivation alone spares short-term 
working memory (Dias and Aggleton, 2000; Izaki et al., 2001), as well as in our case, intermediate-term spatial working memory.

A previous disconnection experiment (Floresco et al., 1997) showed that the interaction between the hippocampus and mPFC was necessary for rats to perform a spatial working memory task with a 30 min delay. However, our results demonstrates that mPFC might not be necessary for an intermediate- or longterm delayed response when only a simple rule (i.e., win shift) is applied to a very small number of trial-unique items (i.e., two different arms per trial in our task). Once a task requires prospective coding (Rainer et al., 1998) of a sequence of several spatial items during an intermediate-term delay [i.e., $30 \mathrm{~min}$ (Floresco et al., 1997)], based on memory of previously coded items (i.e., during sample phase), the interactive communication between the hippocampus and mPFC might become intensified (Floresco et al., 1997), thus resulting in a performance deficit with even one of the communicating partners disabled (Ragozzino et al., 1998). That is, the interaction between the "past" (hippocampus) and the "future" (PFC) may need the intimate PFC-hippocampal interaction especially when those items need to be organized sequentially with a significant amount of delay. Such prospective coding based on retroactive search in memory might be very difficult in our task, because the animals had no prediction, during a delay period, on which side's adjacent arm to a study arm would be chosen as a test arm. Therefore, additional investigation is needed to determine whether the hippocampal-PFC interaction is critical primarily when proactively guidance and/or temporal integration of sequential responses are required (Seamans et al., 1995; Miller, 2000; Fuster, 2001). Furthermore, the relatively long period of delay (i.e., $30 \mathrm{~min}$ ) combined with removal of the rats from the behavioral context, thus necessitating the reinstatement of the context at the time of testing (Redish, 2001), may further recruit the active PFC-hippocampal interaction in the study (Floresco et al., 1997).

Our study provides an essential bridge between the two memory systems that have been emphasized as important in supporting short-term memory (Mishkin, 1978; Kesner and Novak, 1982; Kesner and Adelstein, 1989; Winocur, 1992; Shaw and Aggleton, 1993; Alvarez et al., 1994; Holdstock et al.,1995; Delatour and Gisquet-Verrier, 1996; Granger et al., 1996; Floresco et al., 1997; Wiebe et al., 1997; Rainer et al., 1998; Steele and Morris, 1999; Porter et al., 2000; Clark et al., 2001; Constantinidis et al., 2001; Fuster, 2001; Izaki et al., 2001; Kesner and Hopkins, 2001; Kesner and Rolls, 2001). The current results provide compelling evidence indicating that a mnemonic time window is a critical factor in dissociating the function of the hippocampal system from that of $\mathrm{mPFC}$ in a delayed choice task. That is, the dorsal hippocampus and mPFC appear to process spatial memory in parallel within a short-term range, whereas the dorsal hippocampal function becomes more essential once the critical time window requires spatial memory for a time period exceeding that range.

Given the previously suggested executive and integrative role of PFC (Miller, 2000; Fuster, 2001; Tanji and Hoshi, 2001), our results suggest that PFC may have similar interactions with other brain regions [e.g., inferior temporal cortex (Fuster, 2001; Rolls and Deco, 2002)]. That is, PFC may possess the capability of monitoring virtually the same information processed in other brain regions (e.g., spatial information in the dorsal hippocampus or object-identity information in the inferior temporal cortex) mainly to strategically coordinate multiple systems to achieve a goal. Although the current study showed that the length of delay was one of the critical factors in dissociating parallel coding between the dorsal hippocampus and PFC, what other factors [e.g., familiarity vs novelty (Dolan and Fletcher, 1997; Parkin, 1997; Stern et al., 2001) or prospective vs retrospective coding (Kesner, 1989; Floresco et al., 1997)] also contribute to the interaction between the dorsal hippocampus and PFC (as well as other neocortical structures) needs additional investigation. Because PFC is the brain region that has presumably the most dynamic and richest interactions with other brain regions, it is suggested that understanding the nature of PFC in the context of function of other brain regions is essential (Fuster, 2001).

\section{References}

Alvarez P, Zola-Morgan S, Squire LR (1994) The animal model of human amnesia: long-term memory impaired and short-term memory intact. Proc Natl Acad Sci USA 91:5637-5641.

Clark RE, West AN, Zola SM, Squire LR (2001) Rats with lesions of the hippocampus are impaired on the delayed nonmatching-to-sample task. Hippocampus 11:176-186.

Constantinidis C, Franowicz MN, Goldman-Rakic PS (2001) The sensory nature of mnemonic representation in the primate prefrontal cortex. Nat Neurosci 4:311-316.

Delatour B, Gisquet-Verrier P (1996) Prelimbic cortex specific lesions disrupt delayed-variable response tasks in the rat. Behav Neurosci 110:1282-1298.

Dias R, Aggleton JP (2000) Effects of selective excitotoxic prefrontal lesions on acquisition of nonmatching- and matching-to-place in the T-maze in the rat: differential involvement of the prelimbic-infralimbic and anterior cingulated cortices in providing behavioural flexibility. Eur J Neurosci 12:4457-4466.

Dolan RJ, Fletcher PC (1997) Dissociating prefrontal and hippocampal function in episodic memory coding. Nature 388:582-585.

Eichenbaum H (2000) A cortical-hippocampal system for declarative memory. Nat Rev Neurosci 1:41-50.

Eichenbaum H, Otto T, Cohen NJ (1994) Two functional components of the hippocampal memory system. Behav Brain Sci 17:449-518.

Floresco SB, Seamans JK, Phillips AG (1997) Selective roles for hippocampal, prefrontal cortical, and ventral striatal circuits in radial-arm maze tasks with or without a delay. J Neurosci 17:1880-1890.

Fuster JM (2001) The prefrontal cortex-an update: time is of the essence. Neuron 30:319-333.

Granger R, Wiebe SP, Taketani M, Lynch G (1996) Distinct memory circuits composing the hippocampal region. Hippocampus 6:567-578.

Hampson RE, Jarrard LE, Deadwyler SA (1999) Effects of ibotenate hippocampal and extrahippocampal destruction on delayed-match and -nonmatch-to-sample behavior in rats. J Neurosci 19:1492-1507.

Holdstock JS, Shaw C, Aggleton JP (1995) The performance of amnesic subjects on tests of delayed matching-to-sample and delayed matching-toposition. Neuropsychologia 33:1583-1596.

Izaki Y, Maruki K, Hori K, Nomura M (2001) Effect of rat medial prefrontal cortex temporal inactivation on a delayed alternation task. Neurosci Lett 315:129-132.

Jarrard LE (1993) On the role of the hippocampus in learning and memory in the rat. Behav Neural Biol 60:9-26.

Kesner RP (1989) Retrospective and prospective coding of information: role of the medial prefrontal cortex. Exp Brain Res 74:163-167.

Kesner RP, Adelstein TB (1989) Equivalent spatial location memory deficits in rats with medial septum or hippocampal formation lesions and patients with dementia of the Alzheimer's type. Brain Cogn 9:289-300.

Kesner RP, Hopkins RO (2001) Short-term memory for duration and distance in humans: role of the hippocampus. Neuropsychology 15:58-68.

Kesner RP, Novak JM (1982) Serial position curve in rats: role of the hippocampus. Science 218:173-175.

Kesner RP, Rolls ET (2001) Role of long-term synaptic modification in short-term memory. Hippocampus 11:240-250.

Kim JJ, Baxter MG (2001) Multiple brain-memory systems: the whole does not equal the sum of its parts. Trends Neurosci 24:324-330.

Lee I, Kesner RP (2002) Differential contribution of NMDA receptors in hippocampal subregions to spatial working memory. Nat Neurosci 5:162-168.

Maguire EA, Mummery CJ, Buchel C (2000) Patterns of hippocampal- 
cortical interaction dissociate temporal lobe memory subsystems. Hippocampus 10:475-482.

Martin JH (1991) Autoradiographic estimation of the extent of reversible inactivation produced by microinjection of lidocaine and muscimol in the rat. Neurosci Lett 127:160-164.

Miller EK (2000) The prefrontal cortex and cognitive control. Nat Rev Neurosci 1:59-65.

Mishkin M (1978) Memory in monkeys severely impaired by combined but not separate removal of the amygdala and hippocampus. Nature 273:297-298.

Mishkin M, Appenzeller T (1987) The anatomy of memory. Sci Am 256:80-89.

Moser E, Moser MB, Andersen P (1993) Spatial learning impairment parallels the magnitude of dorsal hippocampal lesions, but is hardly present following ventral lesions. J Neurosci 13:3916-3925.

Moser MB, Moser EI (1998) Functional differentiation in the hippocampus. Hippocampus 8:608-619.

Nadel L (1995) Multiple memory systems: what and why, an update. In: Memory systems (Schacter DL, Tulving E, eds), pp 39-64. Cambridge, MA:MIT.

Nadel L, Samsonovich A, Ryan L, Moscovitch M (2000) Multiple trace theory of human memory: computational, neuroimaging, and neuropsychological results. Hippocampus 10:352-368.

Parkin AJ (1997) Human memory: novelty, association and the brain. Curr Biol 7:R768-R769.

Paxinos G, Watson C (1997) The rat brain in stereotaxic coordinates. New York: Academic.

Porter MC, Burk JA, Mair RG (2000) A comparison of the effects of hippocampal or prefrontal cortical lesions on three versions of delayed nonmatching-to-sample based on positional or spatial cues. Behav Brain Res 109:69-81.

Ragozzino ME, Adams S, Kesner RP (1998) Differential involvement of the dorsal anterior cingulate and prelimbic/infralimbic areas of the rodent prefrontal cortex in spatial working memory. Behav Neurosci 112:293-303.

Ragozzino ME, Detrick S, Kesner RP (2002) The effects of prelimbic and infralimbic lesions on working memory for visual objects in rats. Neurobiol Learn Mem 77:29-43.

Rainer G, Asaad WF, Miller EK (1998) Selective representation of relevant information by neurons in the primate prefrontal cortex. Nature 393:577-579.

Redish AD (2001) The hippocampal debate: are we asking the right questions? Behav Brain Res 14:81-98.

Rolls ET, Deco G (2002) Computational neuroscience of vision. New York: Oxford UP.

Rowe JB, Toni I, Josephs O, Frackowiak RSJ, Passingham RE (2000) The prefrontal cortex: response selection or maintenance within working memory. Science 288:1656-1660.

Scoville WB, Milner B (1957) Loss of recent memory after bilateral hippocampal lesions. J Neurol Neurosurg Psychiatry 20:11-21.

Seamans JK, Floresco SB, Phillips AB (1995) Functional differences between the prelimbic and anterior cingulated regions of the rat prefrontal cortex. Behav Neurosci 109:1063-1073.

Shaw C, Aggleton JP (1993) The effects of fornix and medial prefrontal lesions on delayed non-matching-to-sample by rats. Behav Brain Res 54:91-102.

Squire LR (1992) Memory and the hippocampus: a synthesis from findings with rats, monkeys, and humans. Psychol Rev 99:195-231.

Steele RJ, Morris RGM (1999) Delay-dependent impairment of a matchingto-place task with chronic and intrahippocampal infusion of the NMDAantagonist D-AP5. Hippocampus 9:118-136.

Stern CE, Sherman SJ, Kirchhoff BA, Hasselmo ME (2001) Medial temporal and prefrontal contributions to working memory tasks with novel and familiar stimuli. Hippocampus 11:337-346.

Tanji J, Hoshi E (2001) Behavioral planning in the prefrontal cortex. Curr Opin Neurobiol 11:164-170.

Wiebe SP, Staubli UV, Abros-Ingerson J (1997) Short-term reverberant memory model of hippocampal field CA3. Hippocampus 7:656-665.

Winocur G (1992) A comparison of normal old rats and young adult rats with lesions to the hippocampus or prefrontal cortex on a test of matching-to-sample. Neuropsychologia 9:769-781. 